\title{
Chronic Medical Conditions as Risk Factors for Herpes Zoster
}

\author{
Riduan M. Joesoef, MD, PhD; Rafael Harpaz, MD, MPH; Jessica Leung, MPH; \\ and Stephanie R. Bialek, MD, MPH
}

\begin{abstract}
Objective: To determine the degree to which chronic conditions might contribute to the unexplained burden of herpes zoster.

Methods: We conducted a case-control study using MarketScan data from January 1, 2007, through December 31, 2007, to investigate chronic conditions as risk factors for herpes zoster among persons 20 to 64 years old. Cases were enrollees with a herpes zoster diagnosis (International Classification of Diseases, Ninth Revision, Clinical Modification codes 053.xx), and controls were those without a herpes zoster diagnosis, matched by age groups and insurance plan. We selected 10 chronic conditions based on their prevalence in the general population. We calculated the attributable fraction and created a comorbidity composite score by summing the significant coefficient of regression of chronic conditions. We used logistic regression to evaluate the associations between herpes zoster and chronic conditions.

Results: We identified a total of 59,173 cases and 616,177 controls for the analysis. Risk of herpes zoster was significant for 8 of the 10 study conditions (odds ratios, 1.06-1.52). Herpes zoster risk also increased as a function of comorbidity composite score. The attributable fractions for these 8 significant conditions ranged from $0.24 \%$ to $2.89 \%$. Conclusion: The risk of herpes zoster may be increased in people with chronic conditions. However, this risk may not contribute substantially to the burden of herpes zoster in the population. The causes for most cases of herpes zoster remain unknown.
\end{abstract}

() 2012 Mayo Foundation for Medical Education and Research - Mayo Clin Proc. 2012;87(10):961-967

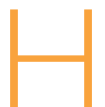

erpes zoster (HZ) is a painful and debilitating illness that affects approximately 1 million individuals annually in the United States. ${ }^{1,2}$ The age-adjusted annual incidence of $\mathrm{HZ}$ has increased from approximately 1.9 per 1000 population in 1993 to 3.8 per 1000 population in 2006. ${ }^{3}$ The incidence, severity of symptoms, and complications of $\mathrm{HZ}$ increase with increasing age. ${ }^{1-4}$ Approximately $30 \%$ of the population will develop $\mathrm{HZ}$ during their lifetime. ${ }^{2}$ Postherpetic neuralgia, a frequent and disabling complication of $\mathrm{HZ}$ in older populations, may persist for years and is frequently refractory to treatment. ${ }^{5}$ A vaccine for $\mathrm{HZ}$ was licensed for use in individuals 60 years or older in the United States in 2006. ${ }^{6,7}$ Although the disease and complications are common among the elderly population, half of the disease incidence is in people younger than 60 years. ${ }^{1,2}$

Herpes zoster is caused by the reactivation of latent varicella zoster virus in the cranial and dorsal root ganglia. ${ }^{1}$ Waning of cell-mediated immunity is thought to be one of the causes of varicella zoster virus reactivation ${ }^{8,9}$ and has been offered as the explanation for why immunosuppressive conditions and advancing age are the most consistently identified risk factors for HZ. However, population-based studies have reported that approximately $90 \%$ of $\mathrm{HZ}$ cases occur among immu- nocompetent patients, for whom risk factors for $\mathrm{HZ}$ are not well characterized. ${ }^{2,10}$

Given the gaps in our understanding regarding the causes of $\mathrm{HZ}$ and the reasons for its increasing incidence, we sought in this study to identify widely prevalent factors that may be unrecognized risk factors for HZ. We explored whether chronic medical conditions, which may themselves be increasing in prevalence, ${ }^{11}$ could be such risk factors, either due to perturbations of varicella zoster virus-specific cell-mediated immunity or through other unknown mechanisms. Although several previous studies have reported possible associations between $\mathrm{HZ}$ and certain chronic conditions (eg, depression, rheumatoid arthritis, and diabetes mellitus), ${ }^{12-19}$ we describe an analysis that did not presume any specific etiologic plausibility; instead, this hypothesis-generating study focused on conditions selected on the basis of prevalence in the general population to identify conditions for which a detected association with $\mathrm{HZ}$ would have potential for substantial attributable population effect.

\section{METHODS}

\section{Data Source}

A case-control study was conducted using commercial claims and encounters data from the 2007 MarketScan databases (MarketScan Database, Thomson
00

From the Centers for Disease Control and Prevention, Atlanta, GA 
Reuters [Healthcare] Inc, Ann Arbor, MI) ${ }^{20}$ The databases include person-specific information from more than 100 large employer-sponsored health plans, state governments, hospitals, health insurance plans, and Medicare. In 2007, the databases included data on approximately 28 million enrollees and more than 500 million medical claims. The databases contain information on patient demographic characteristics, health care professional characteristics, type of insurance plan, dates of services, International Classification of Diseases, Ninth Revision, Clinical Modification (ICD-9-CM) diagnosis codes, and other variables.

\section{Study Population}

The study population consisted of adult enrollees 20 to 64 years old who were continuously enrolled during the study period (January 1, 2007, through December 31, 2007); all variables and outcomes described were from this period. Enrollees were linked across the entire year of available data so that all encounters were captured. We did not study persons 65 years and older because ascertainment of data for the Medicare population was likely different from that in the younger age cohorts. Because we used secondary data that did not have personal identifiers, this study did not require institutional review board approval or informed consent.

\section{Case and Control Selection}

Cases were defined as enrollees with at least one diagnosis of HZ using ICD-9-CM codes of 053.xx recorded in the outpatient files as the primary or secondary diagnosis during the study period. We defined controls as enrollees without any diagnosis of $\mathrm{HZ}$ in the outpatient files during the study period. Age is a strong risk factor for $\mathrm{HZ}$, and type of insurance can serve as a proxy for socioeconomic and health care-seeking behavior. We therefore randomly selected controls by frequency matching for age in 2-year intervals $(20-21,22-23$, and so on to 63-64 years) and type of insurance plan (comprehensive, health maintenance organization, point-ofservice, and preferred provider organization), in a ratio of approximately 1 case to 10 controls.

\section{Validation of Immunosuppressive Conditions as a Risk Factor for $\mathrm{HZ}$}

To determine whether these administrative data could identify unrecognized risk factors for $\mathrm{HZ}$, we first examined whether they could identify established risk factors, such as immunosuppressive conditions and medications. ${ }^{2,21}$ We determined the presence of immunologic disorders, human immunodeficiency virus, AIDS, cancer, organ transplant, and antineoplastic medications defined using ICD-9-CM codes listed in Table 1 and use of immunosuppressive

\begin{tabular}{|c|c|}
\hline Chronic condition & ICD-9-CM code \\
\hline Allergic rhinitis & $477.0-477.9$ \\
\hline Chronic obstructive pulmonary disease & $491 . x \times, 492.8$ \\
\hline Coronary artery disease & 4|| $.8|, 4||.89,4| 3.9,4|4.00-4| 4.05,4|4.2,4| 4.8,4 \mid 4.9$ \\
\hline Depression & $300.4,301.12,311$ \\
\hline Diabetes mellitus & $250.00-250.33,648.00-648.04,250.40-250.93,775.1$ \\
\hline Gout & 274.0 \\
\hline Hyperlipidemia & $272.0-272.4$ \\
\hline Hypertension & $401.0-401.9$ \\
\hline Hypothyroidism & $244.0-244.9$ \\
\hline Osteoarthritis & $715 . x x$ \\
\hline \multicolumn{2}{|c|}{ Immunosuppressive conditions and medications } \\
\hline Antineoplastic medications & E933.I \\
\hline Cancer & 140.xx-172.xx, 174.xx-208.xx, 235.xx-239.xx \\
\hline HIV and AIDS & $079.53,279.10,279.19,795.71,795.8$, V08, 042-044.9 \\
\hline Immunologic disorders & $279.00-279.09,279.11-279.13,279.2-279.9$ \\
\hline Immunosuppressive medications & Therapeutic group $2^{b}$ \\
\hline Organ transplants & 996.80-996.89, V42.0-V42.9, E878-E878.0 \\
\hline
\end{tabular}


medications classified as therapeutic group 21 in the Red Book. ${ }^{22}$ To identify chronic medical conditions that might be unrecognized risk factors for $\mathrm{HZ}$, we excluded all enrollees with these immunosuppressive conditions or medications.

\section{Selection of Chronic Medical Conditions}

We selected 10 chronic medical conditions that are relatively prevalent in the US adult population (allergic rhinitis, chronic obstructive pulmonary disease, coronary artery disease, depression, diabetes mellitus [DM], gout, hyperlipidemia, hypertension, hypothyroidism, and osteoarthritis) regardless of their biologic plausibility of their association with HZ. In instances in which 2 or more prevalent chronic conditions shared pathophysiologic mechanisms (eg, cerebrovascular disease and cardiovascular disease), we only included the more prevalent condition (ie, cardiovascular disease). Once selected, the study conditions were identified by the presence during the study period of one or more ICD-9-CM codes in outpatient claims data in the primary or secondary diagnostic position (Table 1).

We created a comorbidity composite score by summing the significant coefficient of regression $(\beta)$ $(P<.01)$ for chronic conditions in our multivariate analysis and categorizing the summed $\beta$ values as 0 , greater than 0 to less than 0.4 , and 0.4 or greater; these cut points were selected to divide the study population into 3 approximately equally sized groups. For example, if an individual had depression, hypothyroidism, and DM, the comorbidity composite of this individual was the summation of the $\beta$ values for depression, hypothyroidism, and $\mathrm{DM}$. This composite measure assigns weight corresponding to the strength of the association $(\beta)$ between $\mathrm{HZ}$ and each of the chronic conditions. ${ }^{23}$

\section{Statistical Analyses}

We used unconditional logistic regression to evaluate the associations between $\mathrm{HZ}$ and chronic conditions and computed odds ratios and 95\% confidence intervals using SAS statistical software, version 9.2 (SAS Institute Inc, Cary, NC). We ran a logistic model to evaluate the association between $\mathrm{HZ}$ and the 10 chronic conditions adjusted for sex and number of outpatient claims $(<2,3-10,11-20$, $21-30$, or $>30$ claims). On the basis of this model, we ran the final model that excluded from the model the chronic conditions with nonsignificant $\beta$ values. Using the significant $\beta$ values from this model, we computed the comorbidity composite score. We evaluated the association of $\mathrm{HZ}$ with any of the significant chronic conditions and comorbidity composite score adjusted for sex and number of claims. We also conducted $\mathrm{HZ}$ analyses after stratification by age groups (20-40, 41-54, and 55-64 years). To assess the contribution of these chronic conditions to $\mathrm{HZ}$ in the MarketScan study population, we computed the population-attributable fraction, the excess of $\mathrm{HZ}$ cases that could be attributable to the presence of any of these 10 chronic conditions. ${ }^{24}$

\section{RESULTS}

Of the 28,761,500 people in the enrollment files in $2007,20,173,999$ people $(70.1 \%)$ aged 20 to 64 years were enrolled continuously for 12 months. These enrollees form the study population from which 66,921 cases and 663,836 controls were selected. Among cases, $16,196(24.2 \%)$ were aged 20 to 40 years, 25,564 (38.2\%) were aged 41 to 54 years, and 25,162 (37.6\%) were aged 55 to 64 years. Most of the enrollees in the study population were enrolled in preferred provider organizations $(534,295$ [73.1\%]), followed by point-of-service $(78,500[10.7 \%])$, health maintenance organization (98,256 [13.5\%]), and comprehensive plans (19,706 [2.7\%]). Overall, 55,407 study participants (7.6\%) had an immunosuppressive condition and/or were using an immunosuppressive medication. As expected, the risk of $\mathrm{HZ}$ in this group was increased compared with agematched controls, with odds ratios ranging from 1.55 for cancers to 4.43 for human immunodeficiency virus and AIDS and 1.69 for any one of the risk factors (Supplemental Table 1; available online at http://www.mayoclinicproceedings.org).

Our primary study analysis was related to the association of $\mathrm{HZ}$ and chronic conditions. We therefore excluded the 7748 cases and 47,659 controls with immunosuppressive conditions and/or using immunosuppressive medications from that primary analysis to eliminate the possibility that this strong risk factor could introduce residual bias in our results. This exclusion left a total of 59,173 cases with $\mathrm{HZ}$ and 616,177 controls for our primary analysis. Overall, in adjusted analysis, the risk of $\mathrm{HZ}$ was increased by $35 \%$ among women (Table 2). Although the risk of $\mathrm{HZ}$ was increased among persons with a greater number of medical claims (Table 2), for persons with the chronic disease of interest, the median number of claims among cases and controls was similar (Supplemental Table 2; available online at http://www.mayoclinicproceedings.org).

The most prevalent of the chronic conditions among controls and cases were hyperlipidemia, hypertension, and DM. All of the selected chronic medical conditions were more common among cases compared with controls; the adjusted odds ratios ranged from 1.06 for DM to 1.52 for depression (Table 2). These associations were significant for every chronic condition except gout and hypertension. However, the attributable fraction (ie, the excess proportion of $\mathrm{HZ}$ cases that could be attributed) 


\section{TABLE 2. Demographic Factors and Chronic Medical Conditions Among Cases and Controls Aged 20 to 64 Years, MarketScan, 2007a.b}

\begin{tabular}{|c|c|c|c|c|c|}
\hline Demographic factor and comorbidity & $\begin{array}{l}\text { No. }(\%) \text { of controls } \\
\quad(n=6 \mid 6,177)^{c}\end{array}$ & $\begin{array}{l}\text { No. }(\%) \text { of cases } \\
(n=59,173)^{c}\end{array}$ & $\begin{array}{l}\text { Coefficient of } \\
\text { regression }(\beta)\end{array}$ & $\begin{array}{c}\text { Adjusted OR } \\
(95 \% \mathrm{Cl})\end{array}$ & $\begin{array}{l}\text { Attributable } \\
\text { fraction (\%) }\end{array}$ \\
\hline Female & $325,249(52.8)$ & $4 I, 108(62.2)$ & & $1.35(1.32-1.37)$ & \\
\hline \multicolumn{6}{|l|}{ No. of claims } \\
\hline$<2$ & |4|,540 (23.0) & $4460(7.5)$ & & Reference & \\
\hline $3-10$ & 157,676 (25.6) & 19,868 (33.6) & & $3.67(3.54-3.79)$ & \\
\hline $11-20$ & $130,125(21.1)$ & 16,138 (27.3) & & $3.33(3.21-3.44)$ & \\
\hline $21-30$ & $70,890(11.5)$ & 8088 (13.7) & & $2.90(2.80-3.02)$ & \\
\hline$>30$ & I 15,946 (18.8) & $10,619(18.0)$ & & $2.15(2.07-2.23)$ & \\
\hline \multicolumn{6}{|l|}{ Condition } \\
\hline Allergic rhinitis ${ }^{d}$ & $39,080(6.3)$ & $5681(9.6)$ & .34 & $1.41(1.37-1.45)$ & $2.79(2.50-3.07)$ \\
\hline $\mathrm{COPD}^{d}$ & $3946(0.6)$ & $555(0.9)$ & .30 & $1.35(1.23-1.47)$ & $0.24(0.15-0.33)$ \\
\hline$C A D^{d}$ & $21,101(3.4)$ & $2368(4.0)$ & .15 & $1.17(1.11-1.22)$ & $0.58(0.39-0.77)$ \\
\hline Depression ${ }^{d}$ & $|8,29|(3.0)$ & $2922(4.9)$ & .42 & $1.52(1.46-1.58)$ & $1.68(1.49-1.89)$ \\
\hline Diabetes mellitus & $57,874(9.4)$ & $640 \mid(10.8)$ & .06 & $1.06(1.03-1.09)$ & $0.61(0.26-0.96)$ \\
\hline Gout & $1770(0.3)$ & $196(0.3)$ & .27 & & \\
\hline Hyperlipidemia $^{d}$ & | 47,570 (24.0) & $17,190(29.1)$ & .10 & I.11 (1.08-1.13) & $2.89(2.29-3.47)$ \\
\hline Hypertension & 135,257 (22.0) & 14,788 (25.0) & .02 & & \\
\hline Hypothyroidism ${ }^{d}$ & $36,510(5.9)$ & $4874(8.2)$ & .15 & $1.16(1.12-1.20)$ & $1.14(0.83-1.45)$ \\
\hline Osteoarthritis $^{\mathrm{d}}$ & $33,109(5.4)$ & $4133(7.0)$ & .21 & $1.24(1.20-1.28)$ & $1.35(1.10-1.6)$ \\
\hline Any one of these serious conditions ${ }^{\mathrm{e}}$ & $245,755(39.9)$ & $29,397(49.7)$ & & $1.24(1.22-1.27)$ & $9.62(8.42-10.80)$ \\
\hline \multicolumn{6}{|l|}{ Comorbidity composite score $e^{c}$} \\
\hline 0 & $370,422(60.1)$ & $29,776(50.3)$ & & Reference & \\
\hline$>0$ to $<0.4$ & $207,037(33.6)$ & 23,507 (39.7) & & $1.18(1.16-1.20)$ & \\
\hline$\geq 0.4$ & $38,7 \mid 8(6.3)$ & $5890(10.0)$ & & $1.67(1.62-1.72)$ & \\
\hline $\begin{array}{l}{ }^{a} \mathrm{CAD}=\text { coronary artery diseases; } \mathrm{Cl}=\text { confide } \\
\text { ratio. } \\
\text { b Cases and controls were matched for age (2-y } \\
\text { cancers and those using immunosuppressive and } \\
\text { c Excludes the } 47,659 \text { controls and } 7748 \text { cases } \\
{ }^{d} \text { Adjusted for sex, number of claims, and other } \\
\text { e Adjusted for sex and number of claims. }\end{array}$ & $\begin{array}{l}\text { e interval; COPD = chror } \\
\text { ar interval) and type of he } \\
\text { intineoplastic medications } \\
\text { h immunosuppressive con } \\
\text { rious chronic conditions. }\end{array}$ & $\begin{array}{l}\text { c obstructive pulmonar } \\
\text { Ith insurance plan; peo } \\
\text { ere excluded. } \\
\text { itions and/or using imn }\end{array}$ & disease; HIV = hur & $\begin{array}{l}\text { an immunodeficiency } \\
\text { disorders, HIV, AIDS } \\
\text { ations. }\end{array}$ & $\begin{array}{l}\text { ndrome; } \mathrm{OR}=\text { odds } \\
\text { prgan transplants, and }\end{array}$ \\
\hline
\end{tabular}

for each of these conditions was small, ranging from $0.24 \%$ for chronic obstructive pulmonary disease to $2.89 \%$ for hyperlipidemia.

The risk of $\mathrm{HZ}$ increased significantly as a function of the number of chronic conditions $(P=.006)$. The adjusted odds ratio was increased by $18 \%$ among persons with a comorbidity composite score of greater than 0 to less than 0.4 and by $67 \%$ among those with a comorbidity composite of 0.4 or more. This association between $\mathrm{HZ}$ risk and high comorbidity composite score slightly increased with age. No consistent patterns by age groups were observed among individual chronic medical conditions.

\section{DISCUSSION}

Unrecognized risk factors clearly exist for $\mathrm{HZ}$, and it is crucial to identify these risk factors to interpret the epidemiology of HZ, target prevention and treatment strategies, and, in the future, monitor the effect of the $\mathrm{HZ}$ vaccination program. This is the first study, to our knowledge, that has examined several common chronic conditions as possible risk factors for $\mathrm{HZ}$ and assessed the effect of multiple chronic conditions on the risk of developing HZ. Five of the 10 chronic conditions that we found to be associated with HZ (hyperlipidemia, allergic rhinitis, hypothyroidism, osteoarthritis, and chronic obstructive pulmonary disease) have never been previously reported as HZ risk factors. Although we found significant associations between $\mathrm{HZ}$ and 8 of the 10 chronic conditions we investigated, the strength of the associations and their attributable fractions was small. On the basis of this analysis, it seems that chronic conditions do not substantially explain why many persons without recognized risk factors (ie, age 
and immunosuppression) experience $\mathrm{HZ}$ or why the incidence of $\mathrm{HZ}$ is increasing.

Estimation of attributable fraction assumes that associations of chronic conditions and $\mathrm{HZ}$ are causal and that removing chronic conditions has no effect on the distribution of other competing risk factors for $\mathrm{HZ}^{24,25}$ Our estimates of attributable fraction should be interpreted cautiously because these assumptions may not be met, especially on the causal associations of chronic conditions and HZ. However, whether the assumptions for estimating attributable fraction are fully met or not, the findings suggest that the prevalent chronic conditions we investigated do not explain or contribute substantially to the burden or increasing incidence of $\mathrm{HZ}$.

We chose this data source because it allowed for parsimonious investigation of a previously unexplored hypothesis. However, our ability to detect an association between $\mathrm{HZ}$ and prevalent chronic medical conditions may have been limited by our data source and study design. Because this analysis was limited to a single year of claims data, we could only identify cases of $\mathrm{HZ}$ that occurred during that period. It is likely that a portion of persons with chronic diseases included in our control population had had $\mathrm{HZ}$ before the study period, leading to an underestimate of the association between chronic diseases and HZ. The time at which individuals with chronic disease come to medical attention and receive a diagnosis of that condition varies with their health care-seeking behavior. Individuals with chronic medical conditions may be more likely to seek medical care for $\mathrm{HZ}$ or more likely to have it diagnosed during a medical visit because they have more medical conditions and are more inclined to seek medical care. This differential in health careseeking behavior may lead to spurious associations between $\mathrm{HZ}$ and chronic conditions. If those with chronic medical conditions were more inclined to seek medical care for $\mathrm{HZ}$, we would expect on average the number of medical claims among cases with chronic conditions to be higher than among controls with chronic conditions. However, we found that the median number of claims for cases and controls among those with chronic medical conditions was similar. We also adjusted the association between $\mathrm{HZ}$ and chronic conditions with the number of claims. Thus, in our study population, the differential of health care-seeking behavior among cases and controls with chronic conditions would not likely alter the findings of the association between $\mathrm{HZ}$ and chronic conditions.

Finally, each of the chronic conditions we examined included a broad spectrum of disease. It is possible that the risk of $\mathrm{HZ}$ is increased only among persons with longer duration and greater severity of the condition. Claims data do not always allow patients to be categorized by disease severity.

Our findings have several limitations. Ascertainment of HZ through claims data is only possible if patients seek medical care for the condition. Evidence suggests that most persons with $\mathrm{HZ}$ seek medical care. ${ }^{26}$ In addition, there is no mechanism to validate MarketScan diagnostic codes with medical record reviews. However, other studies have used medical record review to report the validity of administrative data in accurately identifying various acute and chronic diagnoses and procedures, including HZ. ${ }^{27-31}$ Our finding that immunosuppressed persons are at increased risk of $\mathrm{HZ}$ provides an additional degree of internal validity for our study methods.

There may be underascertainment of some of the chronic conditions we examined, especially those conditions that are commonly treated with over-the-counter medications, such as allergic rhinitis, without involving a health care encounter. Physicians may not routinely record a patient's chronic conditions on each medical claim, especially if the patient is being seen for an unrelated medical condition. ${ }^{32}$ In addition, the underascertainment may vary, depending on the existence of other chronic conditions. For example, persons with allergic rhinitis and other chronic conditions who access medical care for those other conditions may be more likely to have their allergic rhinitis brought to medical attention or, if they have multiple and more severe chronic conditions, may have the visit coded for the more severe conditions and with the allergic rhinitis not coded at all. The underascertainment of these kinds of chronic conditions may result in a spurious lowering of the estimate of the association with HZ. Because corticosteroid use may not be as fully captured in the MarketScan databases, chronic conditions with heavy use of corticosteroids might spuriously increase the estimate of the association with $\mathrm{HZ}$.

Lastly, the MarketScan databases have limited data on sociodemographic characteristics, which limited our ability to control for potential covariates, such as race and income, in our analysis. The enrollees in the MarketScan databases differ from the general population, and our study does not include persons 65 years and older, but it is unlikely that the magnitude of the association of the study risk factors and $\mathrm{HZ}$ (or the corresponding attributable fractions) would be increased to a substantial degree by age, race, or income.

\section{CONCLUSION}

In this hypothesis-generating study, we identified several relatively prevalent chronic conditions that appear to be associated with an increased risk of $\mathrm{HZ}$, 
including 5 that have not been previously associated with HZ. Although these conditions warrant additional confirmatory study, none of them are likely to account for a significant portion of the burden of $\mathrm{HZ}$ in the general population. These analyses highlight the challenges in investigating risk factors for $\mathrm{HZ}$, a condition whose pathophysiology is poorly understood. The factors that distinguish the almost onethird of immunocompetent persons who experience $\mathrm{HZ}$ during their lifetime from the two-thirds who do not experience $\mathrm{HZ}$ are still to be determined.

\section{SUPPLEMENTAL ONLINE MATERIAL}

Supplemental material can be found online at http://www.mayoclinicproceedings.org.

Abbreviations and Acronyms: $\mathrm{DM}=$ diabetes mellitus; $\mathbf{H Z}=$ herpes zoster; $\mathbf{I C D} \mathbf{- 9 - C M}=$ International Classification of Diseases, Ninth Revision, Clinical Modification

Disclaimer: The findings and conclusions in this report are those of the authors and do not necessarily represent the views of the Centers for Disease Control and Prevention, US Department of Health and Human Services.

Correspondence: Address to Rafael Harpaz, MD, MPH, Centers for Disease Control and Prevention, 1600 Clifton Rd NE, MS A-34, Atlanta, GA 30333 (Rharpaz@cdc.gov).

\section{REFERENCES}

1. Schmader K. Herpes zoster and postherpetic neuralgia in older adults. Clin Geriatr Med. 2007;23(3):615-632.

2. Yawn BP, Saddier P, Wollan PC, St Sauver JL, Kurland MJ, Sy LS. A population-based study of the incidence and complication rates of herpes zoster before zoster vaccine introduction. Mayo Clin Proc. 2007;82( I I): I 34 I - 1349.

3. Leung J, Harpaz R, Molinari NA, Jumaan A, Zhou F. Herpes zoster incidence among insured persons in the United States, 1993-2006: evaluation of impact of varicella vaccination. Clin Infect Dis. 201 1;52(3):332-340

4. Donahue JG, Choo PW, Manson JE, Platt R. The incidence of herpes zoster. Arch Intem Med. 1995; I 55(I 5):1605-1609.

5. Dworkin RH, Schmader KE. Treatment and prevention of postherpetic neuralgia. Clin Infect Dis. 2003;36(7):877-882.

6. Oxman MN, Levin MJ, Johnson GR, et al. A vaccine to prevent herpes zoster and postherpetic neuralgia in older adults. N Engl J Med. 2005;352(22):227I-2284.

7. Harpaz R, Ortega-Sanchez IR, Seward JF; Advisory Committee on Immunization Practices (ACIP), Centers for Disease Control and Prevention (CDC). Prevention of herpes zoster: recommendations of the Advisory Committee on Immunization Practices (ACIP), MMWR Recomm Rep. 2008;57(RR-5): I-30.

8. Cohen JI, Brunell PA, Straus SE, Krause PR. Recent advances in varicella-zoster virus infection. Ann Intern Med. 1999; I30( I I): 922-932.

9. Miller AE. Selective decline in cellular immune response to varicella-zoster in the elderly. Neurology. 1980;30(6):582-587.

10. Grote V, von Kries R, Rosenfeld E, Belohradsky BH, Liese Immunocompetent children account for the majority of complications in childhood herpes zoster. J Infect Dis. 2007; | 96(|0): | 455-1458
11. Medco Health Solution, Inc. Medication Nation: Research finds chronic health problems now afflict more than half of all Americans. http://medco.mediaroom.com/index.php?s=17872\&item $=$ 27977. Accessed August 19, 2011.

12. Heymann AD, Chodick G, Karpati T, et al. Diabetes as a risk factor for herpes zoster infection: results of a populationbased study in Israel. Infection. 2008;36(3):226-230.

13. Smitten AL, Choi HK, Hochberg MC, et al. The risk of herpes zoster in patients with rheumatoid arthritis in the United States and the United Kingdom. Arthritis Rheum. 2007;57(8): |43|-1438.

14. Strangfeld A, Listing J, Herzer P, et al. Risk of herpes zoster in patients with rheumatoid arthritis treated with anti-TNF-alpha agents. JAMA. 2009;30I (7):737-744.

15. Irwin $\mathrm{M}$, Costlow $\mathrm{C}$, Williams $\mathrm{H}$, et al. Cellular immunity to varicella-zoster virus in patients with major depression. J Infect Dis. 1998; 178(suppl I):S104-S108.

16. Livengood JM. The role of stress in the development of herpes zoster and postherpetic neuralgia. Curr Rev Pain. 2000;4(I):7-1।.

17. Schmader K, George LK, Burchett BM, Pieper CF. Racial and psychosocial risk factors for herpes zoster in the elderly. J Infect Dis. 1998;178(suppl I):S67-S70.

18. Dworkin RH, Banks SM. A vulnerability-diathesis-stress model of chronic pain: herpes zoster and the development of postherpetic neuralgia. In: Gatchel RJ, Turk DC, eds. Psychological Factors in Pain: Critical Practices. New York, NY: Guilford Press: 1999:247-269.

19. Wolfe F, Michaud K, Chakravarty EF. Rates and predictors of herpes zoster in patients with rheumatoid arthritis and noninflammatory musculoskeletal disorders. Rheumatology (Oxford). 2006:45( I I): 1 370- 1375.

20. Thompson Healthcare Inc. MarketScan Databases User Guide and Database Dictionary, Commercial and Medicare Supplemental Databases, 2006 Edition. Englewood, CO: Thompson Healthcare Inc; 2007

21. Insinga RP, Itzler RF, Pellissier JM, Saddier P, Nikas AA. The incidence of herpes zoster in a United States administrative database. J Gen Intern Med. 2005;20(8):748-753.

22. Thomson Healthcare. Red Book: Pharmacy's Fundamental Reference. Greenwood Village, CO: Thomson Reuters (Healthcare) Inc; 2008

23. Deyo YA, Cherkin DC, Ciol MA. Adapting a clinical comorbidity index for use with ICD-9-CM administrative databases. J Clin Epidemiol. 1992;45(6):613-619.

24. Rothman KJ, Greenland S, Lash TL. Applications of Stratified Analysis Methods: Modern Epidemiology. 3rd ed. Philadelphia, PA: Lippincott Williams \& Wilkins; 2008:283-302.

25. Levine $\mathrm{B}$. What does the population attributable-fraction mean? http://www.cdc.gov/PCD/issues/2007/jan/06_0091.HTM. Accessed July 19, 2010.

26. Lu PJ, Euler GL, Jumaan AO, Harpaz R. Herpes zoster vaccination among adults aged 60 years or older in the United States, 2007: uptake of the first new vaccine to target seniors. Vaccine. 2009;27(6):882-887

27. Virnig BA, McBean M. Administrative data for public health surveillance and planning. Annu Rev Public Health. 200 I;22:213230.

28. McDonald JR, Zeringue AL, Caplan L, et al. Herpes zoster risk factors in a national cohort of veterans with rheumatoid arthritis. Clin Infect Dis. 2009:48(|0): | 364- |37|. 
29. Bearelly S, Mruthyunjaya P, Tzeng JP, et al. Identification of patients with diabetic macular edema from claims data: a validation study. Arch Ophthalmol. 2008; | 26(7):986-989.

30. Kiyota Y, Schneeweiss S, Glynn RJ, Cannuscio CC, Avorn J, Solomon DH. Accuracy of Medicare claims-based diagnosis of acute myocardial infarction: estimating positive predictive value on the basis of review of hospital records. Am Heart J. 2004; 148(1):99- 104.
31. Jumaan AO, Yu O, Jackson LA, Bohlke K, Galil K, Seward JF. Incidence of herpes zoster, before and after varicella-vaccination-associated decreases in the incidence of varicella, 19922002. J Infect Dis. 2005; 19I (12):2002-2007.

32. Ahmed F, Janes GR, Baron R, Latts LM. Preferred provider organization claims showed high predictive value but missed substantial proportion of adults with high-risk conditions. J Clin Epidemiol. 2005;58(6):624-628. 